\author{
MILITARY TECHNICAL COLLEGE \\ CAIRO - EGYPT
}

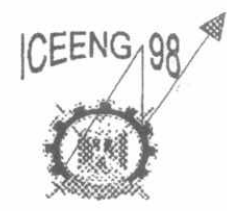

FIRST INTERNATICNAL CONFERENCE

ON ELECT:2ICA: ENGINEERING

MAF.CH 24-26, 1998

\title{
Autopilot Design for \\ a Beam Rider Guided Missile System
}

\author{
Gamal A. El-Sheikh*
}

\begin{abstract}
:
This paper presents some of the fundamental principles involved in tnissile autopilot clesign using the well known classical control approaches and allows a guidance engineer to grasp a sound understanding and knowledge in this field. In addition, the paper provides a robust classic controller for stabilizing the guidance system of a beam rider guided missile in addition to satisfying the performance requirements upon miss distance and demanded normal acceleration. The paper presents the analysis using the analytic approach and the simulation of a simple system from which the concluded remarks are applicable to a more general guidance system. The Paper shows the effects of the sampling period, the compensator time coristants and the guidance gain upon the system performance. The contribution of the paper stems from the easy presentation of a simple classic controller and showing its effect upon the system performance either analytically or through system simulation. The results are obtained using the Guidance systems simulation Toolbox, due the author, under the MATLAB environments.
\end{abstract}

Keywords: Guidance, Automatic Control

\section{1- Introduction}

Guidance is indispensable whenever a missile has to be brought to its pre-designed trajectory or target with high precision. That is, at each instant during the missile flight, the error between the actual trajectory and

- the desired one is measured and shaped to be used for correcting the actual flight path. Thus the guidance system could be, artificially, divided into three subsystems: navigation or measurement, guidance or computation and steering or control. The navigation observes the actual-trajectory parameters during vehicle flight. The guidance system computes the error between the actual and the desired and then shapes these errors to be ready or appropriate for control. The control subsystem executes (responcls to) these guidance commands and generates the lateral forces required to turn the guided vehicle to a new attitude of flight. The point to be remembered here is that a compromise between the conflicting requirements of simple guidance and easy steering has to be worked out (exercised) so as to opt mize the missile system as a whole. In the development of a missile system, experts must be working on each component, trying to make that component do its assigned task with just as little weight, size or space, power, and cost as possible and yet with enough reliability for the purpose.

The field of navigation, guidance and control has grown and given added importance from day to day since the second world war in the forties and their system's applications continue to take on an ever-increasing importance. The guidance system of a guided missile allows and requires that other subsystems or components of the missile be specifically designed and optimized bearing in mind the great interaction or interplay betwesn them. In addition, those working in this field are usually facing many challenges such as information availability, system design complexity, and ever-increasing requirements with the possibility of unstab e or tolerable environments. The stability of the system can be achieved entirely by the careful autopilot design without requiring any particular degree of aerodynamic stability. Under certain assumptions, that could be found in reality, this interaction could be relaxed and consequently the system investigation can be achieved easily.

The guidance and control are characterized by the highly mathematical background nzeded for their synthesis and analysis. In addition, the guidance is a closed loop process in which feedbacks, to be designed, are: utilized to overcome the interferences in the guidance and control systems in addition to satisfying the performance

* Lecturer (B.Sc., M.Sc., Ph.D., MIEEE) in the Guidance Department, Military Technical College, Cairo, Egyrt 
requirements. Therefore, utilizing advanced control techniques such as robust, adartive and intelligent control is indispensable. However, these techniques require high level of mathematical bazkground and experience for selecting the cost functions assigned to the pre-designed performance index.

Therefore, the paper is devoted to present some of the fundamental principles involved in missile autopilot design using the well known classical control approaches. The robustness of the classic controlier is clear in stabilizing the guidance system of a beam rider guided missile in addition to satisfying the performance requirements upon miss distance and demanded normal acceleration. The robustness of the controller stems from the fact that it stabilizes the system and rejects the input disturbance (targef maneuver) with mininium control effort and minimum miss distance. The results are obtained using programs, due the author, under the MATLAB environments.

\section{2- Guidance System Analysis}

\subsection{Motivation for guidance and control}

For military missiles, the targets may be found under the sea, on the sea, on larid, in air or in space and so the launching platforms. Therefore, there are a tremendous number of combinations or engagement scenarios and consequently different classifications of guidance systems and guided missiles. All weapons can be divided into offensive or defensive and the method of intended use into strategic and tactical. Since the weapons became targets for the enemy, their mobility is a main issue and may be extended to many forms on the land, on the sea and in the air, with the basic categories remain unchanged. Therefore, the guided missiles may be classified, according to the purpose and firing or launching and destination, into four general classes: Air-to-Air Missiles (AAM), Air-to-Surface Missiles (ASM), Surface-to-Air Missiles (SAM), Surface-to-Surface Missiles (SSM) [7].

A guided missile has to be equipped with some form of intelligence for achieving the mission with high accuracy. This intelligence is usually termed as guidance of the missile due to which it is narned guided missile. In the case of guided missiles, the instantaneous position and parameters of target flight are estimated upon which guidance or command signals are computed and applied to the missile servo system (autopilot) to correct its flight for intercepting the target with high accuracy and high kill probability. The guidance is a closed loop process in which feedbacks, to be designed, are utilized to overcome the interferences in the guidance and control systems in addition to satisfying the performance requirements. Therefore, utilizing advanced control techniques such as robust, adaptive and intelligent control is indispensable. Consequently, the paper highlights the requirements imposed upon missile autopilot design. The proper guidance of a guided missile, to intercept a target, is the problim of automatic control for positioning the missile on a trajectory along which the missile is to fly in order to hit the target or to miss it at such small distance that the combat charge carried in the warhead destroys hat target. The problem of missile guidance to intercept an enemy target includes several technical disciplines, such as radioloction, computers, telemechanics, rocket engines, aerodynamics of missile flight...etc. The necessity for guiding the missile motion is given by the following points:

1. The need to compensate for the non-standard conditions of the medium, technology, propulsion for the achievement of the desired accuracy of fire at greater ranges

2. The need of reacting on the target motion during the missile flight, and

3. The need for conducting fire behind terrain undulations and artificial obstacles.

According to the guidance and control philosophy utilized to control the missile motion and the location of the guidance computer, there are five types of guidance: Homing guidance, Autonomous guidance, Beam rider guidance, Command guidance and Combined guidance systems [7].

\subsection{Motivation to robust control}

A distinguishing feature of industrial applications, especially the aerospace, is the large envelope of operation in which the process is usually highly nonlinear and has different characteristics from one operating condition to another. In addition, the real environment may change with time (components may age or their parameters may vary with environmental conditions) or operating conditions may vary (load changes, disturbances). Another issue is the model uncertainty since a mathematical representation of a system often involves simplifying assumptions. Nonlinearities are either unknown, and hence unmodeled, or modeled and later ignored to simplify analysis. Different components of systems (actuators, sensors, amplifiers, motors, gears, belts, atc.) are sometimes modelled 
by constant gains, even though they may have dynamics or nonlinearities. Dynamic structures (e.g. robots, aircraft, satellites, missiles) have complicated dynamics in the high frequency region, and these may initially be ignored. Therefore, control systems designed using such simplified models of systems may not work on the real plant in real environments, for which reason the system uncertainties have to be quantified during controller design. Thus the ultimate goal for a control-system designer is to build a system that will work in the real environment and able to withstand all of the above variations.

When modeling an industrial process, either on the basis of physical laws or by system identification, an exact description of the process is never found. There will always be a discrepancy between the actual process and its nominall model. This model error can be represented by an unknown transfer function that indicates the difference between the actual process and the model. The stability of feedback system should be maintained despite model uncertainties. Model uncertainties are generally divided into two categories: structured uncertainty and unstructured uncertainty. Structured uncertainty assumes that the uncertainty is modelled and ranges on bounds for uncertain parameters in the system are available or known. This type of uncertainty is still under irvestigation, due to the complexity of the problem. Unstructured uncertainties assume less knowledge of the system. It is only assumed that the frequency response of the system lies between two bounds. This type of uncertainty comprises two types: additive and multiplicative uncertainty.

The control of uncertain systems can be done using one of two philosophies: the robust control philosophy and adaptive control philosophy. That is, robust control and adaptive control share the same objective which is the control under uncertainty. However, the adaptive control philosophy incorporates some sort of on-line parameter estimation, while a robust control is a fixed controller designed to satisfy performan ze specifications (over a given range of uncertainty. An adaptive controller can learn from experience in the sense that parameters are changed on-line, whereas a robust controller does not learn from past performance. On the other hand, adaptive controllers that perform well in the face of parameteric uncertainty alone are known to be highly sensitive to other types of uncertainties such as unmodeled dynamics and external disturbances. Generally, the adaptive approach is applicable to a wider range of uncertainties, but robust controllers are simple to implement and no time is required to tune the controller with the process variations. Since the adaptation mechanism keeps extracting parameter information in the course of operation, adaptive controllers potentially can provide consistent performance in the face of even very large load variations. Therefore, the trend is to robustify the adaptive controller in order to combine the advantages of both approaches. These trade-offs should be understood well to be able to decide which philosophy is appropriate and which philosophies to combine to gain their advantages and overcome the drawbacks of individuals.

Robust control refers to the design of controllers with low sensitivity to parameter variations, disturbances, unmodeled dynamics and other sources of uncertainty. Because of the nonlinearity and complexity of missile dynamics, the robust control problem is extremely important and has received considerable attention in the research and industry. In robust control, the controller has a fixed structure which yields acceptable performance for a given plant uncertainty. Robust controllers fall into several broad categories such as high-gain, switching or variable-structure (sliding modes and Lyapunov-based guaranteed-stability), and dyramic compensation, where a dynamic compensator is designed to minimize some measure of sensitivity to disturbances and uncertainty. One of these philosophies (dynamic compensators) is the functional analytic method called $H_{\infty}$ optimal control [13]. The trend is to have a nominally linear system, contaminated by nonlinear uncertainties. for which a linear controller is designed to cope with a class of uncertainties. The class of all linear dynamic cimpensators that stabilize the nominally linear system can be computed assuming known bounds on the uncertainty perturbing the linear system.

Classical or conventional controllers are usually designed to satisfy specified requirements for steady state error, transient response, stability margins or closed-loop pole locations. Meeting all these design objectives is usually difficult because of the various trade-offs that have to be made and because of the limitations of the design techniques. For example, classical Bode design achieves satisfactory phase margins and steady state requirements, but the step response characteristics may not be appropriate. One of the main purposes of the ordinary feedback control is to safeguard the performance of the overall system against process parameter variations and disturbances. Most of the real processes involve uncertainty due to measurement noise, load-disturbances corrupting the system output and nonlinear effects such as saturation, backlash and friction in the actuator. Therefore, the general idea of increasing the feedback gain might not succeed in stab:lizing the underlying system and can destabilize it. For example, the problem of steering aerospace vehicles which possess many control subsystems (Pitch controls, Elevator controls, Yaw controls, Thrust controls, ... etc.) should be cptimized to achieve certain safety and performance requirements. However, the classical control techniques can be made 
robust using special approaches for selecting the controller parameters $[2,10,11]$. These approaches constitute in careful selection and implementation and evaluation of the controller within environments close to the actual. Therefore, the objective of the paper, away from the complexity of and the mathematical background required to the modern techniques, is to show how the classical controller can be used with a simplified guided missile model and satisfy the requirements upon good tracking and disturbance rejection.

\subsection{Investigation of missile motion}

The guided missile motion can be investigated utilizing one of three approaches: Kinematic methods, Simplified dynamic methods and Dynamic methods. First approach is the Kinematic methods in which the missile is assumed mass-less point and the equations describing its motion are derived without considering the causes to this motion. Utilizing these methods, it is possible to determine the shape of trajectories and the necessary maneuverability of the missile under the assumption that the velocity of the missile is a known function of time. Second is the simplified dynamic methods in which the missile velocity is continuously determined along the concrete trajectory. The common feature here is the neglection of the missile rotary motion around its c.g. (the missile is considered as a mass point). These methods have two variants according to what extent the constriction limiting the motion of the guided missile is satisfied. Third approach is the dynamic methods in which full dynamic properties of the guided missile and control system are considered. In these methods the whole set of equations describing the missile motion including the equations representing the deviations of control fins and called law of control

\subsection{Simulation of guidance systems}

The first problem faced by the designer of a missile guidance system is that of translating the missile tactical problem into specifications for the guidance system design. A synthesis of the proposed system must be made in order to develop the specifications at a time when only the mathematical expressions which govern the behaviour are known, and those are known only approximately. Simulation of the system by analogue and/or digital computers is employed as an aid to the processes of missile design Complete simulation may give way to partial simulation, as the design progresses, by substituting some of the completed elements of the system for the mathematical expressions previously employed. Therefore, simulation is a continuing aid to designer throughout the duration of the design program When the guidance system has been developed/designed, the behaviour of different equipments is proved by flight tests from which the data are collected utilizing telemetry systems. These data are then evaluated to furnish an additional aid to the designer of the guidance system. This evaluation process and redesign are carried out utilizing the simulation on computers. Therefore, the computers are considered main components of the missile guidance system during design and implementation processes.

Simulation is a process of imitating the behaviour of the actual missile system by the behaviour of some other device easier to construct. This device is the set of physical equations governing the guided missile motion which solved on computers utilizing any of the known and available numerical methods yielding a flexible and reliable tool for system design and analysis. The equations governing the behaviour of the guided missile constitute a set of complicated differential equations involving nonlinearities of many kinds. These nonlinearities may stem from the aerodynamic behaviour or from such mechanical effects as limiting, dead-bands, backlash, and hysteresis effects. Therefore, the solution of these equations can be carried out either through:

- reducing the complexities by considering some simplifying assumptions, keeping in mind that the simplified system should be sufficiently similar to the full system for useful conclusions to be drawn from this simplified system, or

- utilizing the great developments in computation means, such as small size, ligh speed, huge amount of data manipulation, etc.

Therefore, the simulator is an equation solving device and the simulation can be fourd in one of two broad categories as follows:

1. Mathematical simulation (full simulation) in which the entire guided missile problem is represented mathematically by a set of equations to be solved using an appropriate computer. 
This simulation is carried out in steps starting by a simplified version of the modeling and then going gradually towards the full problem with variables close to reality. The simplified phases may constitute the following: longitudinal and lateral aerodynamic stability analysis without and with consideration of the drag equation, stability analysis of the control system, two-dimensional trajuctory analysis without aercidynamic consideration, three-dimensional stability analysis, Separate analysis of launching, midcou se guidance, and homing phases can be carried out. Then, a complete ihree-dimensional trajectory analyses with full regard to aerodynamics and control system performance zan be set out and solved to discover the effects; of different flight parameters in addition to the influence of disturbances and measurement noises upon the performance of the system. This type of simulation has the advantage that the dynamic problems do not have to be solved on a real-time basis i.e. any convenient time scale may be used. However, a full mathematical statement of the problem is required and in case of unknown behaviour for some parts a mathematical expression is assumed leading to some inaccuracies:

2. Tes simulation (partial or physical or hardware-in-loop simulation) in which only part of the guided missile problern is mathematically represented on the simulator while the other parts are inserted as harclware in the ccimputing loop to complete the problem. In this type of simulation the effects due to unknown behaviour of some parts are overcome or avoided. However, the solutions should be carried out on real-time basis to accommodate any actual components in the simulatid loop.

According to the above discussions, the simulation of a guided missile system cian be illastrated in the form of block diagram as shown in Fig. 1. The three-dimensional simulation of a gaided missile problem constitutes a number of aspects such as relative motion, control, aerodynamics, muments, forces, angle of attack and side slip angle computations. From this block diagram it is cleas that the system can be simulated starting from different points. Starting, say, with the missile kinematics and given the motion of the designated target, a relative motion computer determines the deviations from the desired trajectory. These errors are shaped and then applied to the control system which activates the control surfaces and receives inputs from the actual missile motion to allow for maneuvers in progress. The control surface deflection causes aerodynamic forces leading to the required flight course corrections. The angles of attack and sice slip, the angular rate components and the control surface defiections are inputs to aerodynamics while the forces and the moments are the outputs. The missile fore equations are integrated to yield the missile forward velocity and flight path angles or the linear components of the velocity vector in a suitable coordinate system Th: moment equations representing the missile rotation are integrated to yield the comporents of the angtlar rate veotor, then a second integratior yields the missile orientation angles. From the data describing the missife orientation and the missile velcity vector, the angles of attack and side slip are obtained. The actual missile motion parameters are sompared with those of target (desired) vielding errors epresenting the deviation of missile from target and applied to the control system. The control system delivers appropriate signals for control surface deflections to correct the missile flight path and suppress these errors, and so on.

The simulation of a guided missile system can be carried out using differe.t methods depending upon stages of the simulation and on the complications encountered as follows:

1. Analytic methods: in which the behaviour of the servos and airframe in the complete loop is approximated by linear differential equations with constant coefficients. These equations are solved analytically to vield the desired solution.

2. Numerical methods: in which the equations representing the guided missile motion contain some nonlinearities such that the analytic methods can not deal with it. The solution is carried out using hand computation.

3. Automatic methods: in which the equations representing the guided missile motion are so complicated such that the hand computation is inefficient and the computers are more appropriate for obtaining the desired solution. 


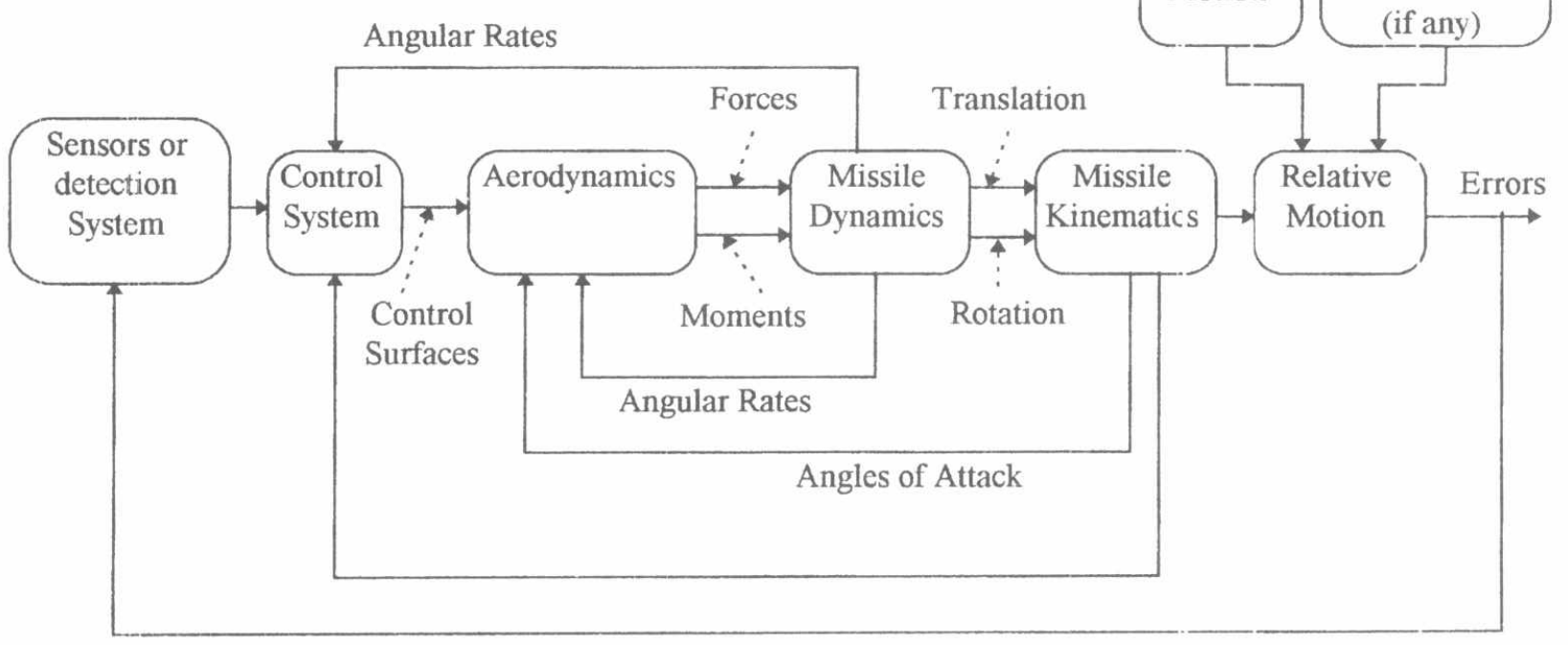

Fig. 1: Simulation of missile guidance system

\section{3- Beam Rider Guidance System}

In a beam rider guidance system an operator or an automatic system in the control point directs the antenna of radio or laser transmitter, producing a beam, toward the assigned direction. The guidance or control signals are formed in the missile so that the missile does not deviate from the center of the bean.. The parent site has to track the designated target continuously during missile flight until interception and the missile navigates itself up the tracking beam by sensing its own location w.r.t. the beam axis. The block diagrain of the beam rider guidance system in pitch plane, with the target tracking and missile guidance loops, is shown in Fig. 2.

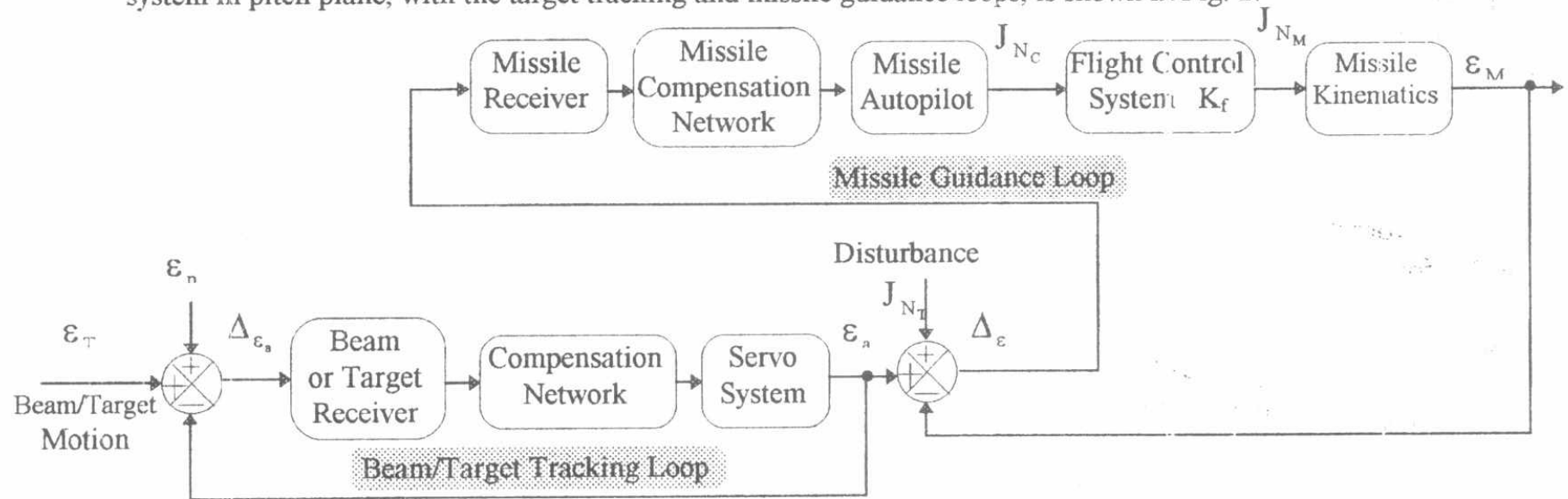

Fig. 2: Beam rider guidance system

The position of a beam rider guided missile and of a target are related to the point of control from which the missile is controlled so as to hit the target. The law of guidance equations are dependent upon the method of guidance used to guide the missile for intercepting the target. That is, the guidance methods limit the direction of vector $\overline{\mathrm{D}}_{\mathrm{M}}$ w.r.t $\overline{\mathrm{D}}_{\mathrm{T}}$ such that they are coincident all the time i.e. $\varepsilon_{M}=\varepsilon_{T}$ and $\sigma_{M}=\sigma_{T}$. Where $\bar{D}_{T}, \bar{D}_{M}$ are, respectively, the target LOS and missile LOS w.r.t. the control point.

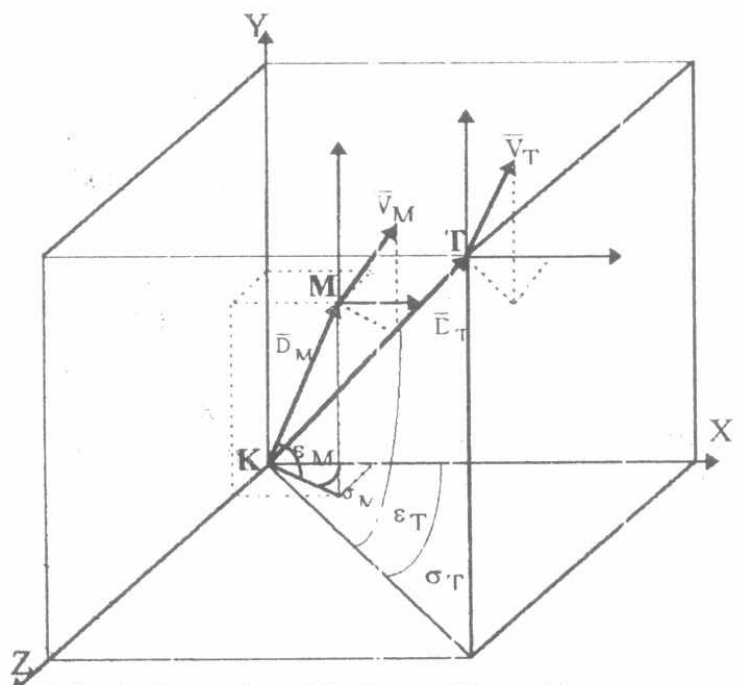

Fig. 3: Geometry of the beam-rider guidance system 


\section{4- Beam Rider Controller Design}

According to the discussion in the previous section, the beam rider guided missile has to fly along a radar/laser beam that is continuously pointed at the designated target. Since the missile has to fly along a moving beam, the guidance commands generated by the computer must be a function of the missile angular deviation from that beam. During the engagement scenario, the beam is always on the target and the missile is always on the beam for achieving the required interception. The guidance equations representing the engagement scenario are nearly identical to those of homing guidance [13]. The geometry of beam rider interception is shown in Fig. 3, where the beam width is assumed zero for simplicity. The objective of the guidance system is to drive the miss distance to zero especially at the end of flight. Thus, the simplest possible implementation of the guidance law for a beam rider guidance system is to make the commanded acceleration proportional to the miss distance or the linea deviation of missile flight from the ideal trajectory i.e.

$$
\mathrm{J}_{\mathrm{N}_{\mathrm{c}}}=\mathrm{N} \eta=\mathrm{ND}_{\mathrm{M}}\left(\varepsilon_{\mathrm{T}}-\varepsilon_{\mathrm{M}}\right)=\mathrm{ND}_{\mathrm{M}} \Delta_{\varepsilon}
$$

where; $\eta$ is the linear deviation of missile flight from the ideal trajectory, $N$ is the guidance gain, $D_{M}$ is the missile LOS, $\Delta_{\varepsilon}$ is the angular missile displacement off the beam. Therefore, the guidance command is proportional to the angular missile displacement off the beam, and consequently off the target.

For the analysis of the system, an analytical linearized model using the small angle approximations has to be found [7]. Considering the linearized model and perfect flight control system, the beam rider guidance loop for the pitch channel can be obtained as shown in Fig. 4. The law of guidance of a beam rider guidance system is represented by the transfer function $\mathrm{G}_{\mathrm{g}}(\mathrm{s})$ that could be selected as static or dynamic element according to the performance requirements. Since, the commanded acceleration is taken to be proportional to the angular missile displacement off the beam the guidance law transfer function has the form: $G_{g}(s)=N$. Therfore, assuming unity flight control system trarisfer $\left(\mathrm{G}_{\mathrm{f}}=1\right)$, the actual missile acceleration to the target elevation can be represented by the following transfer function:

$$
\frac{\mathrm{J}_{\mathrm{N}_{\mathrm{M}}}}{\varepsilon_{\mathrm{T}}}(\mathrm{s})=\frac{\mathrm{ND}_{\mathrm{N}} \mathrm{s}^{2}}{\mathrm{~s}^{2}+\mathrm{N}}
$$

Since the missile lateral \&cceleration is related to the missile elevation by the relation $J_{N_{M}}(s)=D_{M} s^{2} \varepsilon_{M}$, then the actual missile elevation to the target elevation can be represented by the following transfer function:

$8 \quad \frac{\varepsilon_{\mathrm{M}}}{\varepsilon_{\mathrm{T}}}(\mathrm{s})=\frac{\mathrm{N}}{\mathrm{s}^{2}+\mathrm{N}}$

It is clear that the response is oscillatory with natural frequency $\mathrm{N}$. That is, the missile acceleration demanded by the bean! rider guidance system is oscillatory and the miss distance is large. Reducing the value of the guidance gain $\mathrm{N}$ reduces the frequecny of oscillations with increase in the miss distance.

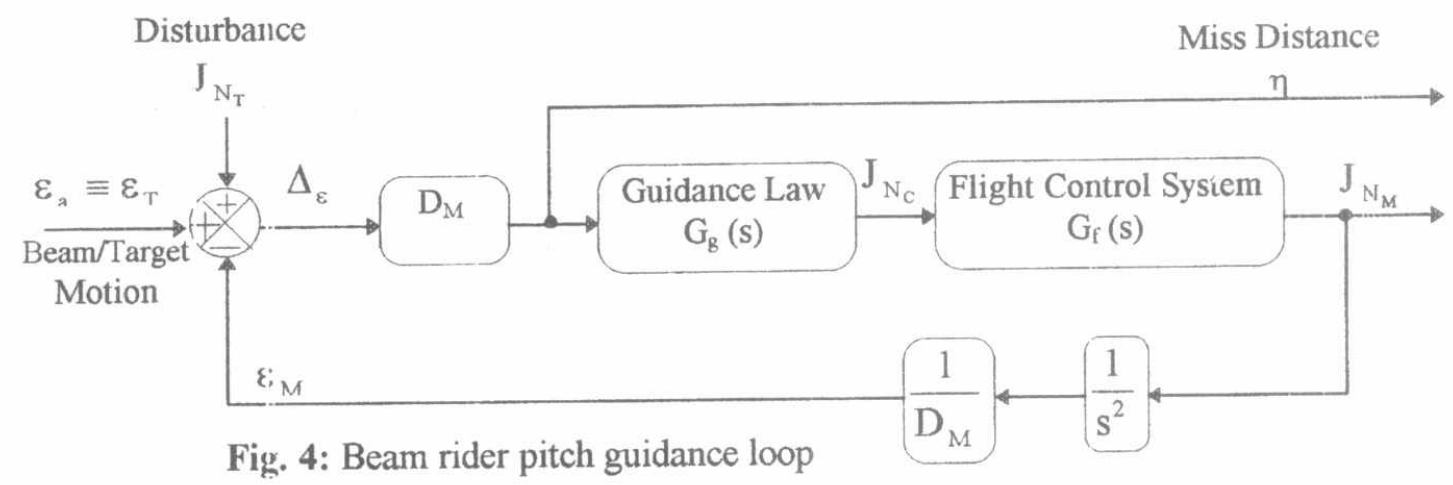

The abov' conclusions can be obtained, as well, by simulating the guidance process of a beam rider guided missile using the equations representing its motion in addition to the law of guidance. As a result for this simulation, the engagement scenario, the commanded acceleration and the miss distance for target speed $V_{T}=0.4[\mathrm{~km} / \mathrm{sec}]$, initial target position is $\left[\mathrm{X}_{\mathrm{T}_{\mathrm{o}}} \mathrm{Y}_{\mathrm{T}_{\mathrm{o}}}\right]=\left[\begin{array}{ll}40 & 20\end{array}\right][\mathrm{km}]$, target maneuver $\mathrm{J}_{\mathrm{N}_{\mathrm{T}}}=3 \mathrm{~g}$, missile speed $\mathrm{V}_{\mathrm{M}}=1.0[\mathrm{~km} / \mathrm{sec}]$, at the initial position $\left[\mathrm{X}_{\mathrm{M}_{\mathrm{o}}} \mathrm{Y}_{\mathrm{M}_{\mathrm{o}}}\right]=\left[\begin{array}{ll}1 & 1\end{array}\right][\mathrm{km}]$ and the guidance gain taken as $\mathrm{N}=5$ are shown in Fig. 5. For stabilizing this system, the guidance law has to be redesigned using any of the control system design approaches. Among these approaches is the simple classical lead-lag compensator whose transfer function has the form 


$$
\mathrm{G}_{\mathrm{g}}(\mathrm{s})=\mathrm{N} \frac{1+\tau_{1} \mathrm{~s}}{1+\tau_{2} \mathrm{~s}}
$$

where the time constants $\tau_{1}$ and $\tau_{2}$ are to be designed $[2,3,6,13]$. A lead-lag network with $\tau_{1}=0.2[\sec ]$ and $\tau_{2}=0.02[\mathrm{sec}]$ is used with the system whose response is shown in Fig. 6 . From th ase figures it is clear how the system became stable and gives good tracking in addition to disturbance rejection capability.
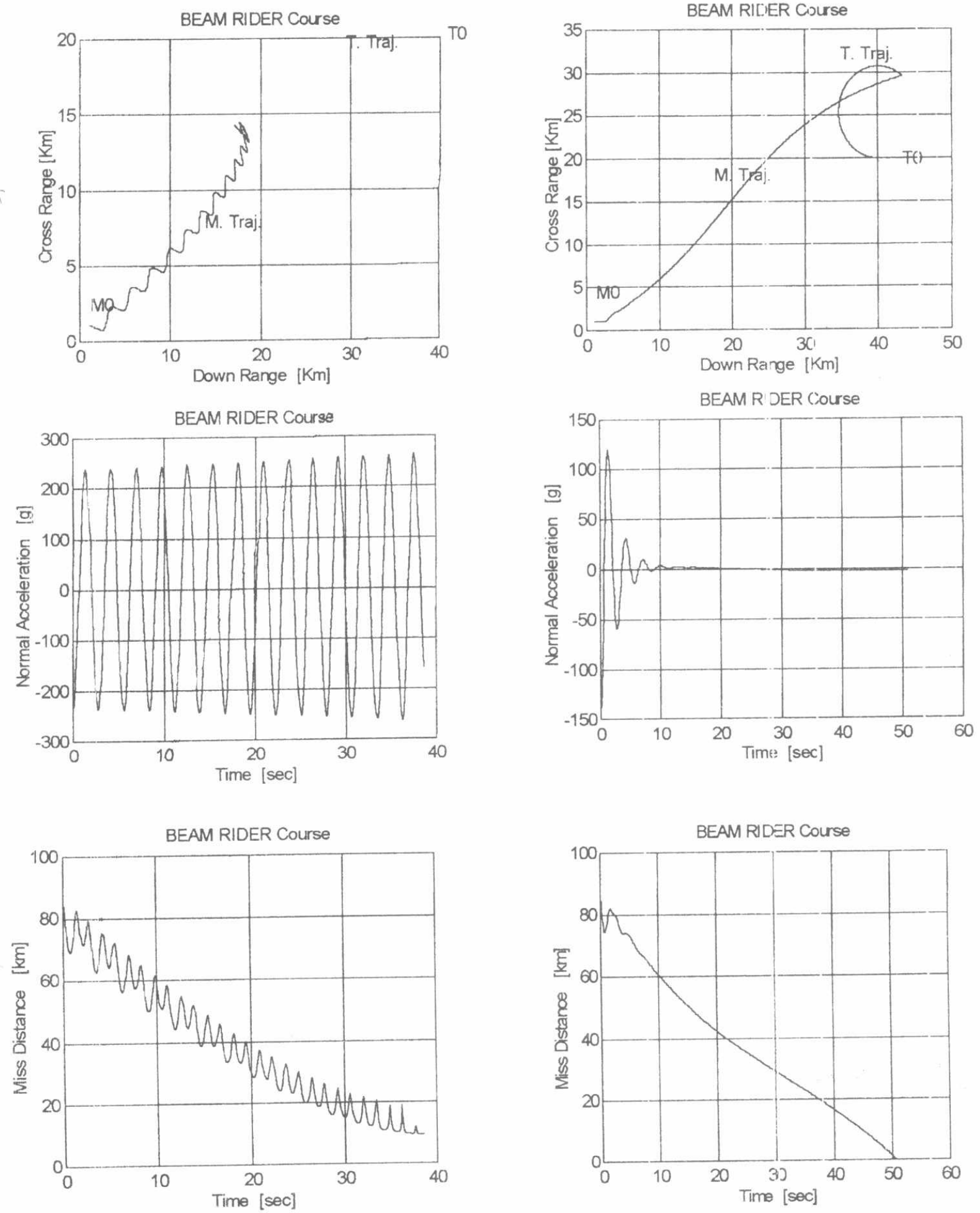

Fig. 5: Uncompensated Beam Rider (a) Engagement scenario, (b) Acceleration and (c) Miss distance

Fig. 6: Compensated Beam Rider (a) Engagernent scenario, (b) Accelerstion and (c) Miss distance 
The simulation process had been run for different sampling periods $T_{s}$ and for different time constants of the compensitor, $\tau_{1}$ and $\tau_{2}$. The results are shown in Tables 1,2 where the appropriate sampling period for this system is $T_{s}=0.08[\mathrm{sec}]$ and the time constants of the compensator are $\tau_{1}=0.2$ [sec] and $\tau_{2}=0.02$ [sec]. These design parameters values gave the minimum miss distance at the end of flight.

\begin{tabular}{|l|l|l|l|l|}
\hline \multicolumn{1}{|c|}{$\tau_{1}[\mathrm{sec}]$} & \multicolumn{1}{|c|}{$\mathrm{T}_{\mathrm{s}}[\mathrm{sec}]$} & Miss Distance $[\mathrm{m}]$ & \multicolumn{1}{|c|}{ Time of Flight [sec] } \\
\hline 0.01 & 0.001 & 0.1 & 151.34 & 68.2 \\
\hline 0.1 & 0.01 & 0.1 & 65.1 & 51.5 \\
\hline 0.2 & 0.02 & 0.1 & 13.27 & 50.8 \\
\hline 0.5 & 0.05 & 0.1 & $12.19218[\mathrm{~km}]$ & 5.2 \\
\hline 0.9 & 0.09 & 0.1 & $120[\mathrm{~km}]$ & \\
\hline
\end{tabular}

Table 1: Effect of the time constants on the miss distance

\begin{tabular}{|l|l|l|l|l|}
\hline \multicolumn{1}{|c|}{$\tau_{1}[\mathrm{sec}]$} & \multicolumn{1}{|c|}{$\mathrm{T}_{\mathrm{s}}[\mathrm{sec}]$} & Miss Distance [m] & \multicolumn{1}{|c|}{ Time of Flight [sec] } \\
\hline \hline 0.2 & 0.02 & 0.05 & 52.44 & 50.8 \\
\hline 0.2 & 0.02 & 0.06 & 23.91 & 50.8 \\
\hline 0.2 & 0.02 & 0.07 & 10.5 & 50.8 \\
\hline 0.2 & 0.02 & 0.08 & 4.51 & 50.8 \\
\hline 0.2 & 0.02 & 0.09 & 8.31 & 50.8 \\
\hline 0.2 & 0.02 & 0.1 & 13.27 & 50.8 \\
\hline 0.2 & 0.02 & 0.2 & 145.55 & 50.8 \\
\hline
\end{tabular}

Table 2: Effect of the sampling period on the miss distance

In addition, the simulation process had been run for different engagement scenarios including short ranges and slow moving targets. using the above sampling period and compensator time constants. The same results are obtained except that the trájectory might be smoother.

\section{Conclusions}

This paper presented some of the fundamental principles involved in missile autopilot design using the well known classical control approaches and allows a guidance engineer to grasp a sound understanding and knowledge in this field. In addition, the paper presented a robust classic controller for stabilizing the guidance system of: a beam rider guided missile in addition to satisfying the performance requirements upon miss distance and demanded normal azceleration. The analysis had carried out using the analytic approach and the simulation of a simple system from which the concluded remarks are applicable to a more general guidance system. The Paper showed the effects of the sampling period, the compensator time constants and the guidance gain upon the system performance. Frorn this analysis the appropriate design parameters values could be obtained and then realized. The novelty of the paper stems from the easy presentation of a simple classic controller and showing its effect upon the system performance either analytically or through system simulation.

\section{References}

[1]Allen E.Puckett, Guicied Missile Engineering, McGraw-hill, 1959.

[2] Astroin, K. J. and B. Wittenmark, Computer Controlled Systems: Theory and Design, Prentice-Hall, 1990.

[3]Blakelock, J.H., Automatic Control of Aircraft and Missiles, Second Edition, John Wiley \& Sons, 1991.

[4]Chin, S.S., Missile Configuration Design, McGraw Hill, 1961.

[5] Cornelisse, J.W., H.F.R.S hoyer, and K.F.Wakker, Rocket Propulsion and Spaceflight Dynamics, Pitman, 1979.

[6] Doyle, J., B.A. Francis, and A.R. Tannenbaum, Feedback Control Theory, Macmillan Publishing Company, 1992.

[7]El-Sheikh, G.A., Theory of Guidance, Lecture Notes, Military Technical College, Cairo, 1987.

[8]Garnell, P., Guided Weapon Control Systems, Pergamon Press, Second Edition, 1980.

[9]Garne:11, P. and D.J. East, Guided Weapon Control Systems, Pergamon Press, First Edition, 1977

[10] Grimble and M.A. Johnson, Robust Control Design: A Tutorial Review, Computing and Control Engineering Journal. November 1991.

[11]Horcwitz, I.M., Syntinesis of Feedback Systems, Academic Press, 1963.

[12]Locke, A.S., Guidance, D. Van Nostrand Company, 1955.

[13]Zarchan, P., Tactica! and Strategic Missile Guidance, Second Edition, AIAA, 1994. 
